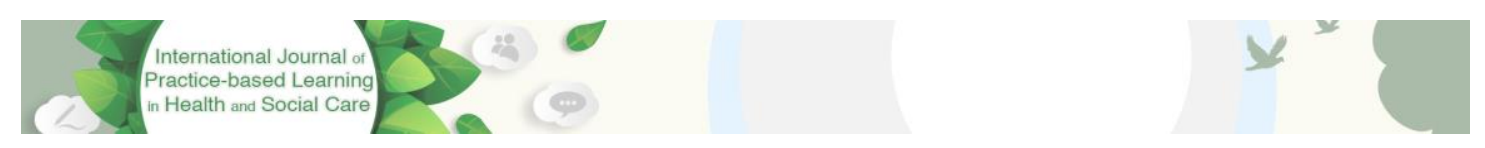

International Journal of Practice-based Learning in Health and Social Care Vol. 4 No 1 July 2016, pages ii-iii

\title{
Editorial Volume 4 Issue 1
}

As the journal enters its fourth year we are delighted that it is regularly attracting an international authorship. It is also beginning to be considered to be a viable outlet for professions lacking a profession-specific peer-reviewed route to publication for educational as opposed to clinical research articles. The great advantage of an interdisciplinary journal is that it shows how the health and social care professions face, and deal with, similar issues and challenges. Many of the ideas are transferable and therefore enhance overall insight into practice-based learning, as well as interdisciplinary understanding. Furthermore, the recurrent interprofessional education theme continues to develop thinking around more explicit moves to bring these professions into closer alignment for the ultimate benefit of collaborative practice.

The current issue is a case in point, reflecting both the generic issues of practice-based learning and an interprofessional theme. The first article from Gillian Nisbet and her colleagues in Sydney, Australia, focuses on a student-led interprofessional initiative, involving medicine, nursing, exercise physiology, diagnostic radiography, occupational therapy, pharmacy, physiotherapy, and speech pathology students. This study, which aimed to investigate the influence of a student-directed experiential interprofessional learning activity, involving both face-to-face communication and social media, found that it had a positive impact on attitudes towards interprofessional learning and working.

Continuing a technology-focused theme, the second article reporting the results of an international project involving academics from the Universities of Sydney, British Columbia and Alberta, explores students' and placement supervisors' perceptions of the potential of telesupervision to support placement learning. Srivalli Nagarajan and the project partners sought to investigate benefits and barriers for language pathology, occupational therapy, physical therapy and exercise physiology students, and clinical education coordinators. Findings suggest that despite limited uptake to date, both students and clinical education coordinators valued, and benefitted from, the regular contact and communication made possible through the use of technology.

The third article, presenting a UK-based study, by Elizabeth Sheils and her colleagues, focuses on the reciprocal relationship between universities and clinical placement supervisors in a clinical psychology context. Exploring this relationship, the study emphasises the perceived benefits of the supervisor role, such as feeling connected to the training course, and the impact of sharing electronic resources on perceptions of connectedness for all stakeholders. The focus for the fourth article by Jim Reeder and Jane Morris, is on the role of paediatric health professionals' as parent educators. This UK-based study, identifies the health professional's role, as a provider of information to parents of children with disabilities, as evolving, from providing information, to supporting the development of parents as learners. The study highlights training needs for developing the skills required to execute this change.

Journal URL: http://e-learning.coventry.ac.uk/ojs/index.php/pblh

(C) 2016 Lynn Clouder. This Open Access article is distributed under the terms of the Creative Commons Attribution Non-Commercial License 4.0 International License

(http://creativecommons.org/licenses/by-nc/4.0/), which permits unrestricted non-commercial use, distribution, and reproduction in any medium, provided the original work is properly cited. 
The final research article by Michael Dunne, a senior speech pathologist based in Australia, and colleagues, reviews the literature on the activities adopted by clinical educators to facilitate reflection in the placement setting. The review explores outcome measures used in current research, and whether contextual factors such as time, safety, mentorship, supervision, and emotional and intellectual support impact on the development of reflection in students. Results suggest that a range of reflective teaching strategies are being used by allied and oral health, nursing, and the medical professions that assist students' critical reflection.

This issue of the journal is concluded with a reflective piece on the author's transition from practitioner to researcher. Sarah Elliott shares her personal journey and experiences of a professional doctorate programme utilising a threshold concepts lens to make sense of her transformed worldview and the impact that this is having on her doctoral studies.

Lynn Clouder, Editor

Coventry University, UK 